\title{
Handling Uncertainty in Recommender Systems under the Belief Function Theory
}

\author{
Raoua Abdelkhalek \\ LARODEC, Institut Supérieur de Gestion de Tunis \\ Université de Tunis, Tunis, Tunisia \\ abdelkhalek_raoua@live.fr
}

\begin{abstract}
Dealing with uncertainty is an important challenge in real world applications including Recommender Systems (RSs). Different kinds of uncertainty can be pervaded at any level throughout the recommendation process, which follows to inaccurate results. The main goal of this research work is to consider RSs under an uncertain framework. We seek for an improvement over the traditional recommendation approaches in order to handle such uncertainty under the belief function theory.
\end{abstract}

\section{Introduction}

Recommender Systems (RSs) [Ricci et al., 2015] have sprung up in the past several years as a powerful tool to cope with the information overload problem. Helping users in their decision making process and guiding them towards relevant items are the main goals of these systems. They tend to predict the users' future preferences and recommendations are then performed accordingly. In fact, a panoply of recommendation approaches has been proposed aiming to enhance the accuracy and the performance of the recommendations. Nonetheless, these approaches are unable to deal with the uncertainty appearing at different phases of the recommendation process, which may seriously affect the RS performance.

Handling uncertainty becomes an active research area and its integration in real world applications becomes an important challenge. That is why, it is fundamental to take it into account. The belief function theory [Dempster, 1968; Shafer, 1976] allows such handling since it represents a rich and flexible framework for reasoning under uncertainty. This theory offers a convenient environment for modeling different types of uncertainty, for combining several pieces of evidence induced from distinct information sources and also for making decisions.

\section{Belief Function Theory}

The Belief function theory [Dempster, 1968; Shafer, 1976] is among the most used theories for reasoning under uncertainty. Assume $\Omega$ be the frame of discernment representing the set of $n$ elementary events such that: $\Omega=$ $\left\{\Omega_{1}, \Omega_{2}, \cdots, \Omega_{n}\right\}$. It contains hypotheses concerning the given problem. The power set of $\Omega$, denoted by $2^{\Omega}$, represents all the possible values that can be taken by each subset of $\Omega$ including singleton hypotheses of $\Omega$, all possible disjunctions of these hypotheses, the empty set $\emptyset$ as well as the entire set $\Omega$. The mapping function $m: 2^{\Omega} \rightarrow[0,1]$ is the basic belief assignment (bba) such that $\sum_{E \subseteq \Omega} m(E)=1 . m(E)$ is called the basic belief mass $(\mathrm{bbm})$ which states the part of belief exactly committed to the event $E$.

The reliability of the piece of evidence can be evaluated through a discounting mechanism as following: $m^{\alpha}(E)=$ $(1-\alpha) \cdot m(E), \forall E \subset \Omega ; m^{\alpha}(\Omega)=\alpha+(1-\alpha) \cdot m(\Omega)$ where $\alpha \in[0,1]$ is the discounting factor.

The fusion of two $b b a$ 's $m_{1}$ and $m_{2}$ derived from two reliable and distinct sources of evidence can be ensured using Dempster's rule of combination defined as:

$\left(m_{1} \oplus m_{2}\right)(E)=k . \sum_{F, G \subseteq \Omega: F \cap G=E} m_{1}(F) \cdot m_{2}(G)$ where
$\left(m_{1} \oplus m_{2}\right)(\emptyset)=0$ and $k^{-1}=1-\sum_{F, G \subseteq \Omega: F \cap G=\emptyset} m_{1}(F)$. $m_{2}(G)$. To make decisions, beliefs can be transformed into a pignistic probability $\operatorname{Bet} P(E)$ and the hypothesis having the maximum value is selected. $\operatorname{Bet} P(E)$ is defined as:

$\operatorname{Bet} P(E)=\sum_{F \subseteq \Omega} \frac{|E \cap F|}{|F|} \frac{m(F)}{(1-m(\emptyset))}$ for all $E \in \Omega$.

\section{Recommender Systems}

The diversity of the information sources and the variety of domain applications gave birth to several recommendation approaches. Collaborative Filtering (CF) [Su and Khoshgoftaar, 2009] is considered among the most promising methods in RSs. This kind of recommender is based on the collaboration between users to filter out relevant items. CF approaches can be either model-based or memory-based. In the model-based category, a model is built to predict ratings based on the entire collection of users' preferences. On the other hand, the memory-based approaches, also refereed to as neighborhoodbased, do not require to learn and maintain a given model to make predictions. They simply compute the similarity between users or items and select the most similar ones for recommendations. There are two main strategies to implement a memory-based CF. The first one, referred to as user-based CF, explores what users with similar tastes have liked. Otherwise, the second one is called item-based CF. It computes similarity between items rather than users to perform recommendations. 


\section{Contributions}

In our research work, we have been notably interested in the $\mathrm{CF}$ recommender and we have successfully made interesting proposals. In a first stage, we have emphasized the relevance of handling uncertainty in neighborhood-based CF approaches, both item-based and user-based ones. We have proposed an evidential item-based CF which incorporates uncertainty in the final predictions based on similar items [Abdelkhalek et al., 2016]. Indeed, the items' ratings have been represented through the belief function tools to measure their degree of uncertainty. Working under the belief function framework, the neighboring items have been considered as distinct pieces of evidence. Their corresponding ratings have been modeled through basic belief assignment ( $b b a$ 's) and aggregated using Dempster's rule of combination in order to perform the final predictions.

Since we cannot admit that these pieces of evidence are fully trusted, we have incorporated a discounting mechanism into the mass functions to quantify the reliability given to each similar item. The key idea was to assign the lowest weights to the items having the highest distances and conversely for the more reliable ones (those having the lowest distances) [Abdelkhalek et al., 2017a].

On the other hand, we have extended the traditional userbased $\mathrm{CF}$ under the belief function framework where similar users have been considered as different pieces of evidence leading to the final prediction [Abdelkhalek et al., 2017c].

In a second stage, we have also exploited the second category of CF namely model-based. In fact, we have conceived a new clustering approach for $\mathrm{CF}$ under the belief function theory where the most similar items have been grouped into different clusters and the predictions are then performed. In our approach, the impact of uncertainty involved during the clusters assignments has been also considered [Abdelkhalek et al., 2017b]. Our obtained experimental results and comparisons with previous works can be found in [Abdelkhalek et al., 2016; 2017a; 2017b; 2017c].

\section{Ongoing-Works and Challenges}

According to the realized work and the achieved results, we keep contributing to research in this area and conceiving new recommendation approaches under an uncertain framework.

Recently, we have proposed to unify both user-based CF and item-based CF approaches based on the belief function framework. Predictions from the K-similar items as well as those of the K-similar users are fused into a unique one using Dempster's rule of combination. The obtained results show that the fusion framework is effective in improving the prediction accuracy of single approaches (user-based and itembased) under certain and uncertain frameworks.

We have also developed a hybrid CF approach that combines both model-based and memory-based CF under the belief function theory. The idea is to build a model from the rating matrix and then performing predictions based on the learned model while taking into account the uncertainty that occurs in the different steps of the recommendation process.

\section{Conclusion and Future Works}

In our research work, we have proposed several CF methods where the uncertainty pervaded in the clusters membership as well as the final prediction process has been considered. Future research directions will focus on conceiving further recommendation approaches under this theory to handle other challenges imposed from the $\mathrm{CF}$ framework such as the cold start and the sparsity problems. Another direction is to examine other types of recommendation approaches beyond the CF. It would be fruitful to develop new evidential hybrid approaches by exploiting different kinds of additional information such as items' features in order to enhance the recommendation performance.

\section{Acknowledgments}

This Ph.D. research project is supervised by Professor Zied Elouedi and Doctor Imen Boukhris. Special thanks to my two supervisors for their valuable support and guidance.

\section{References}

[Abdelkhalek et al., 2016] Raoua Abdelkhalek, Imen Boukhris, and Zied Elouedi. Evidential item-based collaborative filtering. In International Conference on Knowledge Science, Engineering and Management, pages 628-639. Springer, 2016.

[Abdelkhalek et al., 2017a] Raoua Abdelkhalek, Imen Boukhris, and Zied Elouedi. Assessing items reliability for collaborative filtering within the belief function framework. In International Conference on Digital Economy, pages 208-217. Springer, 2017.

[Abdelkhalek et al., 2017b] Raoua Abdelkhalek, Imen Boukhris, and Zied Elouedi. A clustering approach for collaborative filtering under the belief function framework. In European Conference on Symbolic and Quantitative Approaches to Reasoning and Uncertainty, pages 169-178. Springer, 2017.

[Abdelkhalek et al., 2017c] Raoua Abdelkhalek, Imen Boukhris, and Zied Elouedi. A new user-based collaborative filtering under the belief function theory. In International Conference on Industrial, Engineering and Other Applications of Applied Intelligent Systems, pages 315-324. Springer, 2017.

[Dempster, 1968] Arthur P Dempster. A generalization of bayesian inference. In Journal of the Royal Statistical Society, Series B, volume 30, pages 205-247. 1968.

[Ricci et al., 2015] Francesco Ricci, Lior Rokach, and Bracha Shapira. Recommender systems: introduction and challenges. In Recommender systems handbook, pages 134. Springer, 2015.

[Shafer, 1976] Glenn Shafer. A mathematical theory of evidence, volume 42. Princeton university press, 1976.

[Su and Khoshgoftaar, 2009] Xiaoyuan Su and Taghi M Khoshgoftaar. A survey of collaborative filtering techniques. Advances in artificial intelligence, 2009:4, 2009. 